\title{
Inconsistency in Classical Electrodynamics?*
}

\begin{abstract}
F. A. Muller ${ }^{\dagger}$
In a recent issue of this journal, M. Frisch claims to have proven that classical electrodynamics is an inconsistent physical theory. We argue that he has applied classical electrodynamics inconsistently. Frisch also claims that all other classical theories of electromagnetic phenomena, when consistent and in some sense an approximation of classical electrodynamics, are haunted by "serious conceptual problems" that defy resolution. We argue that this claim is based on a partisan if not misleading presentation of theoretical research in classical electrodynamics.
\end{abstract}

1. Introduction. In one of the most provocative papers in the philosophy of science of the last 25 years or so, M. Frisch $(2004,525,530,541)$ first and foremost claims to prove that four assumptions of Classical Electrodynamics (CED) are contradictory. Like "naive set theory" say, CED is nothing short of an inconsistent theory (Inconsistency Claim). Since CED forms the theoretical pillar of our electrified society ever since the end of the nineteenth century and this pillar apparently is logically collapsing, I was relieved to find out that my light switch still worked.

Frisch's $(2004,525,538-539)$ second claim is that all attempts by physicists to revise CED to a theory that is consistent and has in some sense CED as an approximation, give rise to "conceptual problems" that defy resolution or whose solutions ask a price "too high" to pay "in the eyes of most physicists" (Inadequacy Claim). Frisch (2004, 525, 540ff.) then goes on to propose "conditions for the acceptability of inconsistent theories." We shall ignore this proposal because it will turn out to be ill motivated.

*Received November 2005; revised April 2007.

$\dagger$ To contact the author, please write to: Faculty of Philosophy, Erasmus University Rotterdam, Burg. Oudlaan 50, H5-16 Rotterdam, 3062 PA, The Netherlands; e-mail: f.a.muller@fwb.eur.nl; and Department of Physics and Astronomy, Utrecht University, Princetonplein 5, 3584 CC Utrecht, 030-253.3284 Postbus 80000, 3508 TA Utrecht, The Netherlands; e-mail: f.a.muller@phys.uu.nl.

Philosophy of Science, 74 (April 2007) pp. 253-277. 0031-8248/2007/7402-0005\$10.00

Copyright 2007 by the Philosophy of Science Association. All rights reserved. 
Is CED indeed logically flawed and should we honor Frisch for having made this sensational discovery, or is the logic of the proof that has led him to this conclusion flawed? In Section 3, we analyze Frisch's alleged proof and argue that he has applied CED inconsistently. This ought to bring the Inconsistency Claim down. Then in the remainder of this paper, we take a look at three conceptual problems of CED, which according to Frisch defy resolution. We attempt to sketch a faithful picture of how these problems have been handled and are being handled in CED, thereby demonstrating that the account Frisch provides of how these problems are handled in CED is partisan if not downright misleading - an account presumably motivated by gathering support for his Inadequacy Claim. In more detail, in Section 4 we distinguish three kinds of application problems of CED and two classes of CED models; in Section 5, we describe the three conceptual problems that arise in CED; in Sections 6 and 7 we sketch, from the bird's eye point of view, the two main research programs in CED and the various ways in which they handle these problems. This ought to bring Frisch's Inadequacy Claim down. But first of all, in Section 2, we define the theory of CED in order to know exactly what is the object of Frisch's provocative charges.

2. The Postulates of Classical Electrodynamics. First of all, "the language of CED." This language consists of, first, a fraction of English that is sufficient to state and develop the theory and that is unambiguously translatable in other natural languages; second, some parts of mathematics and its accompanying symbols (numbers, real analysis, tensor calculus, differential geometry); third, elementary predicate logic as its deductive apparatus; and fourth, it has the following primitive physical concepts: space, time (or space-time), matter, mass, charge current, force, electromagnetic field, electromagnetic force, and medium. The postulates of CED are the following ones.

Space-Time Postulate. Space-time is mathematically represented by a $3+1$-dimensional, flat, pseudo-metrical Minkowski manifold $\langle\mathcal{M}, \eta\rangle$.

Electromagnetic Field Postulate. The electromagnetic field is mathematically represented by a once-differentiable antisymmetric tensor-valued function $\mathrm{F}$ on $\mathcal{M}$ of rank 2 , the charge-current density by a once-differentiable tensor-valued function $\mathrm{J}$ on $\mathcal{M}$ of rank 1 , and the medium by $\langle\mu, \epsilon\rangle$, where $\mu \in \mathbb{R}$ is a magnetic permeability and $\epsilon \in \mathbb{R}$ is an electric permittivity, such that they obey, for all $x \in \mathcal{M}$ and every component $\alpha, \beta, \gamma \in\{0,1,2,3\}$, the 
homogenous Faraday-Maxwell equations:

$$
\partial_{\alpha} \mathrm{F}_{\beta \gamma}(x)+\partial_{\beta} \mathrm{F}_{\gamma \alpha}(x)+\partial_{\gamma} \mathrm{F}_{\alpha \beta}(x)=0,
$$

and the inhomogeneous Ampère-Maxwell equations:

$$
\partial_{\alpha} \mathrm{F}^{\alpha \beta}(x)=\mu \mathrm{J}^{\beta}(x) .
$$

Electromagnetic Force Postulate. The electromagnetic force is mathematically represented by a tensor-valued function $f_{L}$ of rank 1 that obeys Lorentz's force law:

$$
\mathrm{f}_{\mathrm{L}}^{\alpha}(\mathbf{q}, \mathrm{u})=Q \mathrm{~F}^{\alpha \beta}(\mathbf{q}) \mathrm{u}_{\beta}(\tau),
$$

where $Q \in \mathbb{R}$ is the total charge under consideration, $\mathrm{q}: \tau \mapsto \mathrm{q}(\tau)$ is the worldline of (the center of mass of) the charge distribution $\rho_{\mathrm{c}}(x) \equiv \mathrm{J}_{0}(x) / c$, with affine parameter $\tau$ being the eigentime, and where $\mathrm{u}(\tau) \equiv d \mathrm{q}(\tau) / d \tau$ is the four-velocity of the charge distribution. Dynamical Postulate. Force is mathematically represented by a tensor of rank 1; and the total force $\mathrm{f}_{\mathrm{tot}}$, which may depend on $\mathrm{q}: \tau \mapsto \mathrm{q}(\tau)$ and any of its derivatives, acting on the charge-matter density equals the change in four-momentum $\mathrm{p}(\tau)=m(\tau) \mathrm{u}(\tau)$, where $m(\tau) \in \mathbb{R}$ is the total mass of the charge-matter distribution (usually constant: $m(\tau)=m$ for all $\tau$ ):

$$
\frac{d \mathrm{p}(\tau)}{d \tau}=\mathrm{f}_{\text {tot }},
$$

which we call the Newton-Minkowski Equation.

When the theory of CED is to be defined as a set of structures, or models, rather than as a class of sentences - the elementary deductive closure of the postulates above of CED - then CED is the set of all settheoretical structures of the following type:

$$
\left\langle\mathcal{M}, \eta, \mu, \epsilon, m, Q, \mathrm{~F}, \mathrm{~J}, \mathrm{f}_{\text {tot }}, \mathrm{f}_{\mathrm{L}}\right\rangle,
$$

such that they obey the set-theoretical predicate that consists of the conjunction of the postulates of CED when appropriately translated into the language of set theory (cf. Suppes 2003, 30-33).

When we choose some inertial frame of reference equipped with a Cartesian coordinate system, $\mathbb{R}^{3} \times \mathbb{R}$, on space-time $\langle\mathcal{M}, \eta\rangle$, equations (1) and (2) obtain the following familiar look, which Frisch uses and we shall also mostly use:

$$
\begin{array}{llll}
\nabla \cdot \mathbf{B}(\mathbf{r}, t) & =0, & \nabla \times \mathbf{E}(\mathbf{r}, t)+\partial_{t} \mathbf{B}(\mathbf{r}, t) & =0, \\
\epsilon \nabla \cdot \mathbf{E}(\mathbf{r}, t) & =\rho_{\mathrm{c}}(\mathbf{r}, t), & \nabla \times \mathbf{B}(\mathbf{r}, t)-\epsilon \mu \partial_{t} \mathbf{E}(\mathbf{r}, t)=\mu \mathbf{J}(\mathbf{r}, t),
\end{array}
$$


where $\mathbf{E}(\mathbf{r}, t) \in \mathbb{R}^{3}$ is the electric field, $\mathbf{B}(\mathbf{r}, t) \in \mathbb{R}^{3}$ the magnetic induction field, $\rho(\mathbf{r}, t) \in \mathbb{R}$ the electric charge density, and $\mathbf{J}(\mathbf{r}, t) \in \mathbb{R}^{3}$ the associated current density, all at a space-time point having coordinates $(\mathbf{r}, t) \in$ $\mathbb{R}^{3} \times \mathbb{R}$. The speed of propagation of the electromagnetic fields in the medium is equal to $(\epsilon \mu)^{-1 / 2}$; this speed is in vacuo, which is medium $\left\langle\mu_{0}, \epsilon_{0}\right\rangle$, the speed of light: $c=\left(\epsilon_{0} \mu_{0}\right)^{-1 / 2}$. The relations between the tensor fields in equations (1) and (2) and the three-vector fields in equations (6) are, for every $i, k, l \in\{1,2,3\}$ :

$$
\mathbf{E}_{k}(\mathbf{r}, t)=-c \mathrm{~F}^{0 k}(x) \quad \text { and } \quad \mathbf{B}_{k}(\mathbf{r}, t)=-\frac{1}{2} \varepsilon^{k i l} \mathrm{~F}^{i l}(x),
$$

where $\varepsilon^{k i l}$ is the antisymmetric Lévy-Civita tensor of rank 3, and for the charge and current density:

$$
c \rho_{\mathrm{c}}(\mathbf{r}, t)=\mathrm{J}^{0}(x) \quad \text { and } \quad c \mathbf{J}_{k}(\mathbf{r}, t)=\mathrm{J}^{0}(x) u_{k}(\tau),
$$

where $t=\gamma(u) \tau, \gamma(u) \equiv(1-|\mathbf{u}| / c)^{-1 / 2}$, and $\mathbf{u}(t) \equiv \dot{\mathbf{q}}(t)$ is the particle's three-velocity. The integral of the charge-matter density $\rho(\mathbf{r}, t)$ over $\mathbb{R}^{3}$ is required to be equal to 1 for all $t \in \mathbb{R}$, so that the charge density is $\rho_{\mathrm{c}}=Q \rho$ and the matter density $\rho_{\mathrm{m}}=m \rho$.

Frisch considers a point particle carrying electric charge $Q=e$. Say it has worldline $\mathbf{q}: t \mapsto \mathbf{q}(t)$. Then the charge-matter density at $(\mathbf{r}, t)$ is Dirac's delta functional $\delta(\mathbf{r}-\mathbf{q}(t))$. The charge and current density become functionals too:

$$
\rho_{\mathrm{c}}(\mathbf{r}, t)=e \delta(\mathbf{r}-\mathbf{q}(t)) \quad \text { and } \quad \mathbf{J}(\mathbf{r}, t)=\rho_{\mathrm{c}}(\mathbf{r}, t) \mathbf{u}(t)=e \delta(\mathbf{r}-\mathbf{q}(t)) \mathbf{u}(t) .
$$

The electromagnetic force three-vector $\mathbf{F}_{\mathrm{L}}$ on the point charge in an electromagnetic field $\langle\mathbf{E}, \mathbf{B}\rangle$ is governed by Lorentz's force law (3):

$$
\mathbf{F}_{\mathbf{L}}(\mathbf{q}(t), \mathbf{u}(t))=e \mathbf{E}(\mathbf{q}(t), t)+e \frac{\mathbf{u}(t)}{c} \times \mathbf{B}(\mathbf{q}(t), t) .
$$

At this point an announcement is in order. The fact that we are dealing with a point particle is not the source of the contradiction that Frisch claims to deduce; his deduction would also have worked for extended charge densities. In this light it is puzzling why Frisch did not ab initio present the argument for an extended charge density, because it would have saved him the trouble of writing extensively about the point particles. Of course, point particles do give rise to a problem (cf. Section 5). But the issue of point particles is not germane to Frisch's proof. We move on.

Frisch considers Newton's law of motion “in the absence of non-elec- 
tromagnetic forces" for a point particle; in this case $\mathbf{F}_{\mathrm{tot}}=\mathbf{F}_{\mathrm{L}}$ :

$$
\dot{\mathbf{p}}_{\mathrm{nr}}(t)=m \mathbf{a}(t)=\mathbf{F}_{\mathrm{L}}(\mathbf{q}(t), \mathbf{u}(t)),
$$

where $\mathbf{p}_{\mathrm{nr}}(t)=m \mathbf{u}(t)$, the particle's nonrelativistic linear momentum, and $\mathbf{a}(t) \equiv \dot{\mathbf{u}}(t)$, the particle's acceleration three-vector. Newton's nonrelativistic equation of motion in relativistic Minkowski space-time? Frisch $(2004,528)$ clarifies: "Hence, when I speak of Newton's laws in this paper, I intend this to include their relativistic generalizations"; which is the Newton-Minkowski equation (4); its three-vector part in the situation under consideration is:

$$
\dot{\mathbf{p}}(t)=\gamma(u) m \mathbf{a}(t)=\mathbf{F}_{\mathrm{L}}(\mathbf{q}(t), \mathbf{u}(t)),
$$

where $\mathbf{p}=\gamma(u) m \mathbf{u}$ is the particle's relativistic momentum three-vector part of the four-vector $p$.

3. Analysis of Frisch's Inconsistency Proof. The four assumptions of CED that Frisch $(2004,525,530)$ brands "internally inconsistent" are the following ones.

(i) There are discrete, finitely charged accelerating particles.

(ii) Charged particles function as sources of electromagnetic fields in acccord with the Maxwell equations (6).

(iii) Charged particles obey Newton's law of motion (11).

(iv) Energy is conserved in particle-field interactions.

How do these four assumptions (i)-(iv) relate to the postulates of CED? First, if assumption (i) is supposed to express that there are CED models in the domain of theoretical discourse of CED — which is inside that of set theory-having a point-particle charge and current density (9), then assumption (i) is a provable mathematical theorem and not an assumption of CED. If assumption (i) is supposed to be an existential statement about what there is in physical reality, then (i) is not part of CED and then (i) expresses a realist attitude toward CED at most. Assumption (i) can then be rejected if the conjunction of CED and (i) leads to a contradiction. This sounds a bit cheap, however. Let us therefore adopt the aforementioned interpretation of (i), which renders assumption (i) harmless.

Second, assumption (ii) is the Electromagnetic Field Postulate and (iii) is a consequence of the Dynamical Postulate and the Electromagnetic Force Postulate under the assumption that the Lorentz force is the only force acting on the point particle $\left(f_{\text {tot }}=f_{L}\right)$. The Space-Time Postulate is not mentioned by Frisch but we shall take it to be tacitly assumed.

Third, there is no mentioning of energy in the postulates of CED, which raises the question how (iv) can be an assumption of CED, as Frisch claims. 
The standard is to prove conservation of energy and momentum on the basis of the symmetries of the dynamical equations of the theory under consideration. In order to get such a proof going in CED, one needs a definition of the energy-momentum tensor of a combined system of particles and fields. The history of CED teaches us that it can be accomplished, although this is far from a straightforward affair as prima facie may seem (cf. Rohrlich 1970; Landau and Lifshitz 1975, 77-80; Schwinger 1978). If one chooses for a Hamiltonian or for a Lagrangean approach to CED, energy conservation is automatically guaranteed as a consequence of the time-translation symmetry-an instance of Noether's Theorem. Hence it stands beyond disputation that (iv) is better seen as a consequence of the postulates of CED rather than as an assumption of CED.

To summarize, we are prepared to accept that the conjunction of CED and the statement ' $f_{\text {tot }}=\mathrm{f}_{\mathrm{L}}$ ' is inconsistent if assumptions (i)-(iv) are inconsistent. With this in position, we move on to Frisch's argument.

Frisch $(2004,530)$ begins his proof by asserting that the following expression "follows from the Maxwell equations in conjunction with the standard way of defining the energy associated." He comes up with the instantaneous power of the electromagnetic field emitted by a moving charged particle at $\mathbf{q}(t)$ having acceleration $\mathbf{a}(t)$ :

$$
P_{\mathrm{Lr}}(t)=\frac{2 e^{2}}{3 c^{3}}|\mathbf{a}(t)|^{2}>0
$$

What Frisch says is not quite true.

Formula (13) is the so-called Larmor formula; it is the result of a nonrelativistic approximation in an adiabatic limit (slowly varying fields), using the Sommerfeld radiation boundary condition. The formula that does follow from the Maxwell equations, in the adiabatic limit, by integrating the Poynting vector $\mathbf{S}(\mathbf{r}, t) \equiv(\mathbf{E}(\mathbf{r}, t) \times \mathbf{B}(\mathbf{r}, t)) / \mu_{0}$ of the electromagnetic field (representing the energy flux of it) over the surface of a retarded sphere surrounding the charge ("the standard way of defining the energy"), is Liénard's formula (Jackson 1975, 660):

$$
P_{\mathrm{Ln}}(t)=\frac{2 e^{2}}{3 c^{3}} \gamma^{6}(|\mathbf{u}(t)|)\left(|\mathbf{a}(t)|^{2}-c^{-2}|\mathbf{u}(t) \times \mathbf{a}(t)|^{2}\right)>0 .
$$

So we have discovered two other tacit assumptions which do not follow from the postulates of CED:

(v) The electromagnetic fields vary slowly (adiabatic limit).

(vi) Sommerfeld radiation boundary condition.

Since this adiabatic limit is not important for the purpose of the current 
section, and we gladly accept that sources are sources and not sinks of radiation energy, we accept (v) and (vi), and we accept that the conjunction of CED and $f_{\text {tot }}=f_{L}$ is inconsistent if assumptions (i)-(vi) are inconsistent.

The energy radiated by the moving charge, via its emitted electromagnetic field, often called the self-field, $\mathbf{E}_{\text {self }}$ and $\mathbf{B}_{\text {self }}$, during time interval $\left[t_{A}, t_{B}\right]$, then is positive:

$$
E_{\mathrm{rad}}(A, B)=\int_{t_{A}}^{t_{B}} P(t) d t>0,
$$

irrespective of whether we take Larmor's formula (13) or Liénard's formula (14) as the integrand $P(t)$ of integral (15). Newton's law of motion (11) and "the definition of external work done on a charge imply that the work on the charge is equal to the change in energy of the charge" (Frisch 2004, 530):

$$
W_{\mathrm{ext}}(A, B)=\int_{A}^{B} \mathbf{F}_{\mathrm{ext}}(\mathbf{r}, t) \cdot d \mathbf{l}=E_{\mathrm{kin}}(B)-E_{\mathrm{kin}}(A),
$$

where in this case $\mathbf{F}_{\text {ext }}=\mathbf{F}_{\mathrm{L}}$, and the line integral is taken along the worldline $\mathbf{q}(t)$ of the charge from point $A$ to point $B$ on it. "But for energy to be conserved, that is for assumption (vi) to hold, the energy of the charge at $t_{B}$ should be less by the amount of the energy radiated $E_{\mathrm{rad}}(A, B)$ than the sum of the energy at $t_{A}$ and the work done on the charge" (Frisch 2004, 530):

$$
E_{\text {kin }}(B)=E_{\text {kin }}(A)+W_{\text {ext }}(A, B)-E_{\text {rad }}(A, B) .
$$

From equalities (16) and (17) it follows that

$$
E_{\mathrm{rad}}(A, B)=0,
$$

in contradiction to inequality (15). Quod erat demonstrandum?

Yes, but demonstrated it is not. Here begins our criticism of Frisch's Inconsistency Claim. When Frisch $(2004,528)$ asserts that "in the absence of non-electromagnetic forces" equation (11) is Newton's law of motion, he is simply in error. Newton's law of motion says that the change in momentum of a physical object having constant mass $m$ equals the total force $\mathbf{F}_{\text {tot }}$ exerted on the physical object (Goldstein 1980, 1; Suppes 2003, 319-321):

$$
\dot{\mathbf{p}}(t)=\mathbf{F}_{\text {tot }} .
$$

The total force $\mathbf{F}_{\text {tot }}$ is usually broken up in two summands: the resulting external and the resulting internal force acting on the physical object under 
consideration (Goldstein 1980, 5; Suppes 2003, 319-321):

$$
\mathbf{F}_{\mathrm{tot}}=\mathbf{F}_{\mathrm{ext}}+\mathbf{F}_{\mathrm{int}} .
$$

In the situation under consideration, the emitted self-field $\left\langle\mathbf{E}_{\text {self }}, \mathbf{B}_{\text {self }}\right\rangle$ of the charge - earlier assumed by Frisch not to vanish, in order to derive that $E_{\mathrm{rad}}(A, B)>0(15)$ - exerts an additional, internal force on the charge, the self-force $\left(\mathbf{F}_{\text {int }}=\mathbf{F}_{\text {self }}\right.$ ), and the external force is the Lorentz force $\left(\mathbf{F}_{\text {ext }}=\mathbf{F}_{\mathrm{L}}\right)$; then Newton's law (19) becomes:

$$
\dot{\mathbf{p}}(t)=\mathbf{F}_{\mathrm{L}}+\mathbf{F}_{\text {self }} \text {. }
$$

In other words, "in the absence of non-electromagnetic forces" we have a total electromagnetic force $\mathbf{F}_{\text {tot }}=\mathbf{F}_{\mathrm{L}}+\mathbf{F}_{\text {self }}$.

Consequently the total work $W_{\text {tot }}(A, B)$ done on the charge results from this total force:

$$
W_{\mathrm{tot}}(A, B)=\int_{A}^{B} \mathbf{F}_{\mathrm{tot}}(\mathbf{r}, t) \cdot d \mathbf{l}=E_{\mathrm{kin}}(B)-E_{\mathrm{kin}}(A) .
$$

We can generally subdivide $W_{\text {tot }}$ in an amount $W_{\text {ext }}$, performed by the external force $\mathbf{F}_{\text {ext }}$ (which is here the Lorentz force $\mathbf{F}_{\mathrm{L}}$ ), and an amount $W_{\text {self }}$, performed by the self-force $\mathbf{F}_{\text {self }}$; in our case:

$$
W_{\mathrm{tot}}(A, B)=W_{\mathrm{ext}}(A, B)+W_{\mathrm{self}}(A, B) .
$$

From equations (17), (22), and (23), we deduce that

$$
E_{\text {rad }}(A, B)=-W_{\text {self }}(A, B) .
$$

No inconsistency follows from equation (24) and inequality (15). On the contrary, they are perfectly consistent.

What Frisch takes into account at the beginning, which is the radiated electromagnetic field by the moving charge $-\mathbf{E}_{\text {self }} \neq 0 \neq \mathbf{B}_{\text {self }}$, so that $\mathbf{F}_{\text {self }} \neq 0$, from which it follows that along $\mathbf{q}$ from $A$ to $B, W_{\text {self }}(A, B) \neq$ 0 , so that $E_{\mathrm{rad}}(A, B) \neq 0$ - he ignores in the next step- $\mathbf{E}_{\text {self }}=0=\mathbf{B}_{\text {self }}$, so that $W_{\text {tot }}=W_{\text {ext }}$, from which it follows that $\mathbf{F}_{\text {self }}=0$ and therefore $W_{\text {self }}(A, B)=E_{\text {rad }}(A, B)=0$. In this fashion one can prove every (set of assumptions extracted from some) theory to be inconsistent: take something into account in the beginning and ignore it in the next step. This is a recipe for logical disaster. What we are dealing with here is an inconsistent application of a theory, not a proof of the inconsistency of the applied theory.

Once again, the logical structure of Frisch's argument consists in correctly arguing, on the basis of CED, for the following two conditional 
statements:

(Step 1) if $\mathbf{E}_{\text {self }} \neq 0 \neq \mathbf{B}_{\text {self }}$, then $E_{\text {rad }}(A, B) \neq 0(15)$;
and
(Step 2) if $\mathbf{E}_{\text {self }}=0=\mathbf{B}_{\text {self }}$, then $E_{\text {self }}(A, B)=0$ (18).

Then he tacitly assumes both antecedents of step 1 and step 2, deduces by modus ponendo ponens both consequents, which amounts to deducing a contradiction. But, then, one is inclined to remark, if both contradictory antecedents are assumed, we do not need the arguments establishing step 1 and step 2 (25) anymore, because then we already have a contradiction by $\wedge$-introduction! The flaw in concluding the inconsistency of CED does not lie in CED but in assuming two contradictory assumptions: the antecedents of step 1 and step 2 (25). No matter in the context of which scientific theory one follows this procedure, one is bound to end up in contradictions. This surely is the easiest recipe for logical disaster: make two assumptions one of which is the negation of the other.

Here ends our criticism of Frisch's Inconsistency Claim. We conclude that Frisch has not proved that assumptions (i)-(iv) - or more precisely (i)-(vi) - are inconsistent, in contradiction to what he has claimed.

A final remark. What does rigorously follow from the postulates of CED is the antecedent of step 1 (25). But as we shall see in the next section, in most descriptions or explanations of electromagnetic phenomena in the domain of CED, the self-fields are ignored, that is, the antecedent of step 2 is taken aboard as an approximation or idealization. Specifically, since only in some ultrarelativistic situations $\mathbf{F}_{\text {self }}$ is of an order of magnitude comparable to that of $\mathbf{F}_{\mathrm{L}}$ and in all other situations $\mathbf{F}_{\text {self }}$ is much smaller (for the synchrotron, see Shen 1972; Lieu 1987), $\mathbf{F}_{\text {self }}$ can be safely neglected when it comes to solving the equation of motion (21): $\mathbf{F}_{\text {self }} \approx 0$. Strictly speaking, one should then write ' $W_{\text {tot }} \approx W_{\text {ext }}$ ', rather than ' $W_{\text {tot }}=W_{\text {ext }}$ ', so that it is immediately clear that no contradiction ensues. In fact, physicists are notoriously sloppy in this respect: a majority of the exact equality signs $(=)$ in most physics papers, articles, and books means approximate equality $(\approx)$. Specifically, in Frisch's argument, ' $=$ ' should be replaced with ' $\approx$ ' in formulae (13) - because derived from $\mathbf{F}_{\text {self }} \neq 0$ - and in formulae (14), (16), and (18)-because derived from $\mathbf{F}_{\text {self }} \approx 0$.

4. Application Problems. Frisch's argument $(2004,529)$ originates in the classical electrodynamic description of the behavior of charged particles in a synchroton accelerator, which he sees as an illustration of the inconsistent descriptions that CED generates. The problem of how to describe the entire behavior of this physical system correctly does not fall in either one of two classes of application problems that CED handles (application problem here being to find a description or explanation of any given 
phenomenon that falls in the domain of CED by means of some CED model):

A-Problems. The charge densities are specified (the worldlines of their centers of mass in space-time and, hence, their current densities): the electromagnetic fields are calculated by solving the Maxwell equations (6) - mathematically the most general problem is to solve a system of 12 coupled first-order partial differential equations.

B-Problems. The electromagnetic fields are specified (and hence the Lorentz forces [10] acting on the charge densities): the worldlines of the (centers of mass of the) charge and current densities are calculated by solving the Newton-Minkowski equation of motion (4) - mathematically the most general problem is to solve a system of three at least second-order partial differential equations.

In A-Problems, the charges are seen as the sources of electromagnetic fields, carrying energy and momentum, but their specified worldlines are not corrected for by the self-force that the emitted fields exercise on the charges, whereas in B-Problems the specified electromagnetic fields are not corrected for the fields emitted by the moving charges. Although it is a blunt fact that every single one of the overwhelming majority of electromagnetic phenomena can be treated as an A- or a B-Problem "with negligible error," as Jackson puts it in his tome $(1975,781)$, both remain approximations in that the self-force is neglected $\left(\mathbf{F}_{\text {self }} \approx 0\right)$. In a completely theoretically satisfactory treatment of these phenomena, self-effects should be taken into account. Jackson writes $(1975,781)$ that "a completely satisfactory treatment of the reaction effects of radiation does not exist" and provides a twofold explanation of this state of affairs.

First, self-effects are not needed to describe or explain the phenomena inside the domain of CED: with negligible error every problem solved by CED can be classified as being either an A-Problem or a B-Problem.

Second, the microscopic behavior of point-like charges, such as electrons, lies altogether outside the domain of CED; it belongs to the realm of quantum physics, where classical physics generally breaks down anyway.

Nevertheless Jackson then goes on to discuss quite a few attempts to deal with self-effects, some of which are as old as CED itself. Let us state the problem in full generality.

Suppose an external electromagnetic field $\left\langle\mathbf{E}_{\text {ext }}, \mathbf{B}_{\text {ext }}\right\rangle$ and some charge density $\rho_{\mathrm{c}}$ with associated current density $\mathbf{J}$ are given. These fields exert an external force $\mathbf{F}_{\mathrm{L}}$ on $\rho_{\mathrm{c}}$ via Lorentz's force law (10). The moving charge density and current emit an electromagnetic self-field $\left\langle\mathbf{E}_{\text {self }}, \mathbf{B}_{\text {self }}\right\rangle$, which is a solution of the Maxwell equations (6) - provided of course that the 
worldline $\mathbf{q}$ of $\rho_{\mathrm{c}}$ does not belong to the class of radiationless motions for point charges (see Pearle 1977, 1978). These self-fields exert an internal force on the charge, the self-force $\mathbf{F}_{\text {self }}$, again via Lorentz's force law (10). The motion of the center of mass of the charge, having worldline $\mathbf{q}(t)$ and linear momentum $\mathbf{p}(t)$, is the solution of the following equation of motion (19):

$$
\dot{\mathbf{p}}(t)=\mathbf{F}_{\mathrm{L}}+\mathbf{F}_{\text {self }} .
$$

These are

C-Problems. Solve the system of 15 coupled partial differential equations (6) and (26) for $\mathbf{q}, \mathbf{E}=\mathbf{E}_{\text {ext }}+\mathbf{E}_{\text {self }}$, and $\mathbf{B}=\mathbf{B}_{\text {ext }}+\mathbf{B}_{\text {self }}$, using Lorentz's force law (10).

Since this system of coupled differential equations rarely admits a solution "in closed form," approximations and idealizations are mandatory to get anywhere. There is a variety of ways of how to approximate what and how to idealize what. Detailed models are called for. Small wonder that there is not a single account of self-effects available but there is a multitude of accounts, each of which rely on different approximations and different idealizations - and this is not quite the same thing as there being no account at all.

Let $\mathcal{A B}$ be called the class of CED models that solve A- or B-Problems, and $\mathcal{C}$ the class of the ones that solve $\mathrm{C}$-Problems. Then models in $\mathcal{A B}$ neglect self-effects whereas models in $\mathcal{C}$ take them into account. Jackson $(1975,781-782)$ provides conditions in terms of typical energy values and times of the physical system under consideration in order to decide whether the problem is $\mathrm{A}, \mathrm{B}$, or $\mathrm{C}$, hence whether the model is in $\mathcal{A B}$ or $\mathcal{C}$. When we take CED to be a class of models (5), then $\mathcal{A B}$ and $\mathcal{C}$ subdivide it, albeit vaguely.

The CED description of the synchroton accelerator, discussed by Frisch (2004, 529), is atypical in that the problem to provide a description or explanation of what happens in this device is divided into two separate problems: an A-Problem and a B-Problem. B-Problem: the circular orbit of the accelerated electron is obtained from a specified homogeneous magnetic induction field by solving the equation of motion, neglecting $\mathbf{F}_{\text {self }}$ because it is estimated to be about 10 orders of magnitude smaller than the external Lorentz force $\mathbf{F}_{\mathrm{L}}$ (see Lieu 1987). A-Problem: the emitted radiation of the electron is studied by considering its specified circular trajectory and solving the inhomogeneous Maxwell equations from the Liénard-Wiechert potentials, in order to determine the nature of the emitted "synchrotron radiation." In the synchrotron accelerator one compensates for the loss of the energy of the electron by making it periodically 
pass an electric potential difference, which changes polarity twice during one revolution. Hence the fact that the accelerated electrons function as sources of radiation is taken fully into account in the design and operation of the synchrotron, because their loss of kinetic energy is appreciable and is compensated for, but $\mathbf{F}_{\text {self }}$ resulting from these emitted fields is ignored because it is far too small in comparison to the external Lorentz force to have any appreciable effect on the circular orbit of the charges. No relevant C-Problem is considered.

In summary, two application problems of a different kind (A and B) yet both pertaining to a single situation, that is, an electron in a synchrotron accelerator, and both making the same assumption $(\mathbf{F} \approx 0)$, are solved by CED. This method of making different approximations dependent on which quantitative problem pertaining to a single phenomenon one attempts to solve is generic in physics in particular and in science in general, we submit. If this generic way of doing things in science were sufficient to pronounce the applied theory inconsistent, then we ridiculously would have to pronounce every single scientific theory inconsistent.

Frisch admittedly mentions one of the attempts to solve a C-Problem as a possible way out of his own alleged inconsistency proof but rejects this attempt because it suffers from conceptual problems $(2004,538)$, about which more in Section 5. But this means that, on Frisch's own terms, the conclusion that CED is inconsistent already is a non sequitur, because what he apparently has established is a Dilemma:

\section{Inconsistency $\vee$ Conceptual Problems.}

Faced with Dilemma (27), we are free to choose what seems to be the horn of lesser evil, namely, the right horn of Conceptual Problems. Logic does not compel us to choose for the left horn of Inconsistency (27). On the contrary, logic compels us to choose for Conceptual Problems (27), due to the following elimination rule for absurdum $(\perp)$ :

$$
\perp \vee \varphi \vdash \varphi,
$$

where $\varphi$ is a sentence variable. Hence, even if Frisch had proved that the six assumptions (i)-(vi) lead to a contradiction, his claim to have proved the inconsistency of CED would still be a non sequitur. Rescued from contradictions we could then pay all our attention to the Conceptual Problems, which is what we actually do next.

5. Conceptual Problems. When Frisch (2004, 537-540) draws his "serious conceptual problems" into the limelight, he is reporting problems of CED models of charges that are extremely well known in physics.

I. The electromagnetic potential energy of a point charge diverges. 
II. The occurrence of pre-accelerations: a charge accelerating before the force begins to act on it.

III. The occurrence of self-accelerations: a charge accelerating further after the force has stopped acting on it.

These conceptual problems are related to the ABC-classification of application problems (Section 4) as follows: problem I arises rigorously whenever one uses point particles, no matter which kind of problem one is trying to solve (A, B, or C). Problems II and III arise only in the process of solving some C-Problem by means of some approximation.

The time scales involved in pre-acceleration effects are extremely small, and therefore these effects are generally not seen as a very serious problem (II). Problems I and III are seen as more pressing. Notice that a point particle that keeps accelerating will keep emitting radiation energy too, which makes it effectively a source of infinite energy. Since it has an infinite amount of energy according to problem I, there is consistency even among the infinities! In the current section we briefly expand on these problems and then give a glimpse of how physicists have handled them and are handling them wholly within CED.

Let us begin with problem I. Every physicist at the end of the nineteenth century knew that the electrostatic energy of a point charge diverges. Let us see how this problem arises rigorously from the postulates of CED.

We consider a point charge in vacuo having electric charge $e$ and worldline $\mathbf{q}: t \mapsto \mathbf{q}(t)$ in the Cartesian coordinate system of some reference frame on Minkowski space-time. Define the retarded time $t_{\mathrm{ret}}$ as the time at which the worldline $\mathbf{q}$ crosses the backward light cone with apex at $(\mathbf{r}, t)$ :

$$
c t_{\text {ret }}=c t-\left|\mathbf{r}-\mathbf{q}\left(t_{\text {ret }}\right)\right| .
$$

Introducing the unit vector $\mathbf{n} \in \mathbb{R}^{3}$ such that

$$
\left|\mathbf{r}-\mathbf{q}\left(t_{\text {ret }}\right)\right| \mathbf{n}=\mathbf{r}-\mathbf{q}\left(t_{\text {ret }}\right),
$$

permits us to write for the retarded electric Lénard-Wiechert field of the moving point charge (Spohn 2004, 12):

$$
\begin{aligned}
\mathbf{E}_{0}(\mathbf{r}, t)= & \frac{e}{4 \pi \epsilon_{0}} \frac{\delta\left(t-t_{\mathrm{ret}}\right)}{(1-\mathbf{u}(t) \cdot \mathbf{n} / c)^{3}}\left[\frac{\left(1-|\mathbf{u}(t)|^{2} / c^{2}\right)(\mathbf{n}-\mathbf{u}(t) / c)}{|\mathbf{r}-\mathbf{q}(t)|^{2}}\right. \\
& \left.+\frac{\mathbf{n} \times((\mathbf{n}-\mathbf{u}(t) / c) \times \mathbf{a}(t) / c)}{|\mathbf{r}-\mathbf{q}(t)|}\right] .
\end{aligned}
$$

The first term is the "near field": it falls off with the square of the distance of the source and always remains with the particle. The second term is the "far field": it falls off with the distance and is proportional 
to the acceleration of the point charge and therefore vanishes when the particle moves uniformly; it dominates at large distances. The field $\mathbf{E}_{0}$ diverges at $\mathbf{r}=\mathbf{q}(t)$ as $|\mathbf{r}-\mathbf{q}(t)|^{-2}$. The associated electrostatic potential $\phi_{0}: \mathbb{R}^{3} \rightarrow \mathbb{R}$ can be calculated from: $\mathbf{E}_{0}(\mathbf{r}, t)=-\nabla \phi_{0}(\mathbf{r})$. The accompanying magnetic induction field $\mathbf{B}_{0}(\mathbf{r}, t)$ is determined by: $c \mathbf{B}_{0}(\mathbf{r}, t)=$ $\mathbf{n} \times \mathbf{E}_{0}(\mathbf{r}, t)$, which is the relativistic generalization of the magnetic law of Biot and Savart. When the point charge is at rest, so that the velocity and the acceleration vanish $(\mathbf{u}(t)=0$ and $\mathbf{a}(t)=0)$, the field reduces to the retarded Coulomb field $\mathbf{E}_{0}^{\text {rest; }}$; it drops out of the "near field" in (31):

$$
\mathbf{E}_{0}^{\text {rest }}(\mathbf{r}, t)=\frac{e}{4 \pi \epsilon_{0}} \frac{\delta\left(t-t_{\mathrm{ret}}\right) \mathbf{n}}{|\mathbf{r}-\mathbf{q}(t)|^{2}} \quad \text { and } \quad \mathbf{B}_{0}^{\text {rest }}(\mathbf{r}, t)=0 .
$$

The energy density $u_{0}: \mathbb{R}^{4} \rightarrow \mathbb{R}$ of this electromagnetic field yields:

$$
\begin{aligned}
u_{0}(\mathbf{r}, t) & =\frac{1}{2} \delta\left(t-t_{\mathrm{ret}}\right)\left(\mu_{0}^{-1}\left|\mathbf{B}_{0}^{\mathrm{rest}}(\mathbf{r}, t)\right|^{2}+\epsilon_{0}\left|\mathbf{E}_{0}^{\mathrm{rest}}(\mathbf{r}, t)\right|^{2}\right) \\
& =\frac{e^{2}}{32 \pi^{2} \epsilon_{0}} \frac{\delta\left(t-t_{\mathrm{ret}}\right)}{|\mathbf{r}-\mathbf{q}(t)|^{4}} .
\end{aligned}
$$

In order to obtain the electrostatic energy of a point charge at rest at the center of a ball of radius $R=\left|\mathbf{r}-\mathbf{q}\left(t_{\text {ret }}\right)\right|$, one integrates $u_{0}$ (33) over it. Going to spherical coordinates, $(r, \Omega) \in \mathbb{R}^{+} \times[0,4 \pi)$, one obtains for the total electrostatic potential energy for a point charge at rest:

$$
\begin{aligned}
U_{\mathrm{pot}}\left(\mathbf{E}_{0}^{\mathrm{rest}}\right) & =\lim _{R \uparrow \infty} \lim _{d \downarrow 0} \int_{0}^{4 \pi} d \Omega \int_{d}^{R} u_{0}(r, \Omega) r^{2} d r \\
& =-\frac{e^{2}}{8 \pi \epsilon_{0}}\left(\lim _{R \uparrow \infty} \frac{1}{R}-\lim _{d \downarrow 0} \frac{1}{d}\right) \leadsto+\infty .
\end{aligned}
$$

Notice that when we integrate over all of space save some minute hole of radius $d>0$ around the point charge, no matter how small, the integral does not diverge: it is the contribution of the near field when we take the point-particle limit ( $d \downarrow 0$ ) that blows $U_{\text {pot }}\left(\mathbf{E}_{0}^{\text {rest }}\right)$ up. (Something similar happens for the potential energy of Newton's gravity potential; see Rohrlich 1999.)

Mathematically speaking, $U_{\text {pot }}$ is a functional, sending every electromagnetic field $\langle\mathbf{E}, \mathbf{B}\rangle$ to a real number, called its total potential energy, $U_{\text {pot }}(\mathbf{E}, \mathbf{B})$. The functional here only depends on the electric field and takes the familiar form of a definite integral (34). When this integral diverges, the conclusion is that $\mathbf{E}_{0}^{\text {rest }}$ (31) does not belong to the domain of $U_{\text {pot }}$, which is some subset of the class of all integrable (and differentiable) functions 
from $\mathbb{R}^{3}$ to $\mathbb{R}$. Any conclusion from (34) to the effect that CED is inconsistent would be illicit, just as illicit as when we would conclude the inconsistency of Real Analysis from applying function $x \mapsto 1 / x$ to 0 (one is forbidden to apply it to 0 because 0 is not in the domain of $x \mapsto 1 / x$ ). Some physicists have made this illicit move. One example is Feynman $(1964,28-1)$, who says in this context the following about point particles: "The concept of simple charged particles and the electromagnetic field are in some way inconsistent." Another example is from the celebrated textbook duo Landau and Lifshitz $(1975,90)$, who state more precisely in this context: "Since the occurrence of the physically meaningless infinite self-energy of the elementary particle is related to the fact that such a particle must be considered as point-like, we can conclude that electrodynamics as a logically closed physical theory presents internal contradictions when we go to sufficiently small distances." For the sake of emphasis, Feynman and Landau and Lifshitz-illicitly—speak about "inconsistent" and "contradictions," respectively, because of the diverging potential energy of a point particle - this has nothing to do with Frisch's alleged inconsistency proof.

Physically speaking, if $U_{\text {pot }}$ of a point charge at rest diverges (34), then so does its inertia according to Einstein's universal mass-energy relation $E=m c^{2}$; this means that a point charge would have infinite inertia and therefore would never respond to forces acting on it. Point charges are immovable objects. Since electrons - the very first elementary particles, introduced by J. J. Thompson and H. A. Lorentz, the founding fathers of elementary particle physics - do move and can be accelerated, the proper conclusion to draw is that according to CED electrons are not point particles.

Furthermore, when we want to describe the joint evolution of a point charge and the electromagnetic fields, the mathematics breaks down because the solution (32) of the Maxwell equations is singular at precisely the points where we need to know the Lorentz force: on the worldline $t \mapsto \mathbf{q}(t)$ of the charge.

The proper general conclusion to draw is that point particles fall outside the domain of CED: the models of point particles turn out to be not models of CED, appearances to the contrary notwithstanding. Hence there is a serious problem for constructing models of (presumably nonexistent) point particles: the argument leading to the diverging potential energy (34) literally is a reductio ad infinitum of the statement that CED does contain models of point particles. Surprisingly, CED does not contain these models. To repeat, this is not to say that CED is an inconsistent theory.

The natural move is to construct models of charged particles having a spatially extended charge distribution-remember what we remarked just 
below formula (34). Another move is to change the definition of $U_{\text {pot }}$; an example is the "Bopp integral," expounded by Feynman (1964, § 28-5). An altogether different move is to insist that models of point particles fit within CED, by pointing out an unwarranted tacit assumption in the argument above that has led us to the conclusion that point particles fall outside the domain of CED, and then to begin afresh without that assumption. These two moves correspond to a broad classification of theoretical research in CED in two research programs, which we call the Extension Program and the Renormalization Program, respectivelywhere it is to be remarked that syntheses of both programs are around too.

So much for problem I. Problems II and III are generated by approximate solutions of C-Problems in both research programs. To these programs we turn next.

6. The Renormalization Program. Historically the Extension Program started around the turn of the twentieth century, with Max Abraham's semi-relativistic and Lorentz's fully relativistic model of the electron by a spherically symmetric extended charge distribution (see Rohrlich 1965, 8-25; Spohn 2004, 33-36). After the rise of quantum physics, these classical models threatened to fade away into oblivion, but the threat never really materialized and, in fact, recently these models have reentered center stage of theoretical research in CED. The other program in CED is the Renormalization Program; it was initiated in 1938 by Dirac, who insisted on working with point particles. We outline the Renormalization Program in the current section and the Extension Program in the next section.

The conclusion that point charges are immovable objects relies on an attractive assumption that was part and parcel of the so-called Electromagnetic Worldview. This worldview loomed large among physicists around the turn of the twentieth century and was promulgated in particular by Wilhelm Wien, Lorentz, and Abraham. That attractive assumption is that all matter is of electromagnetic origin and thus somehow inertia is a function of its charge and emitted fields via their potential energy: $m=f\left(e, U_{\text {pot }}\right)$. (Remember that in those days the elementary particles were exhausted by electrons and atoms.) If therefore the potential energy $U_{\text {pot }}$ diverges, then so does its mass $m$-provided one assumes that $f$ is an increasing function of $U_{\text {pot }}$ for fixed $e$ without horizontal asymptote. But as Dirac $(1938,148)$ emphasized, since the discovery of the neutron in 1932, which is a massive $(m>0)$ and electrically neutral particle, this assumption has lost its attraction. The neutron deals a considerable blow to the Electromagnetic Worldview. About electrons Dirac $(1938,155)$ supposed that "there is an infinite negative mass at its center such that, when subtracted from the infinite positive mass of the surrounding Coulomb 
field, the difference is well defined and is just equal to $m$." Hence we write:

$$
m_{\text {exp }}=m_{\mathrm{f}}-m_{\mathrm{b}},
$$

where $m_{\exp } \equiv m$ is the experimentally determined mass, $m_{\mathrm{f}}$ the field or electromagnetic mass, and $m_{\mathrm{b}}$ the bare mass. The field mass $m_{\mathrm{f}}$ can in turn be broken up in a longitudinal and transversal component (first done by J. J. Thompson; see Lorentz 1909, 38-39). Anyhow, problem I, then, is solved.

Enter Frisch (2004, 538), who dismisses this solution in a single line on the basis of nothing more than a pejorative metaphor: "sweeping the infinity of the self-fields under the rug."

I beg to disagree. Actually two infinities are hauled from under the rug and put on display on top of the rug; then we let them annihilate each other in order to be left with a single finite quantity. This is the spectacle that Dirac presents. We are not saying that these violent clashes of infinities belong to the most endearing spectacles in theoretical physics to watch, but we are saying that no mathematical laws need be broken in such a clash. After all, the difference between two diverging series may very well converge. As often, mathematically speaking Dirac lives dangerously, but he lives. Dirac $(1938,149,155)$ asserted that in the light of quantum physics, his CED model of the electron "is hardly plausible," but he added that as long as "we have a reasonable mathematical schema" and the reasonable mathematical schema is "in agreement with well-established principles, such as the principle of relativity and the conservation of energy and momentum," one should not object to this CED model. Dirac then derived, as an approximate solution of the self-problem, an equation of motion for point charges that was derived about 30 years earlier by Lorentz and Abraham for spatially extended charge distributions. This is the celebrated Lorentz-Dirac equation (see below).

Let us first mention what Jackson $(1975,784)$ calls the Abraham-Lorentz equation (historically terribly inaccurate but we follow suit for want of terminology):

$$
d_{\tau} \mathrm{p}(\tau) \approx \mathrm{f}_{\mathrm{L}}+\mathrm{f}_{\text {Schott }}^{\alpha},
$$

where $f_{L}$ is the external Lorentz four-force (3), and $f_{\text {Schott }}$ is the "Schott term"-after the physicist G. A. Schott (see Rohrlich 1965, 150; 2000) for their explicit expressions. The Schott term $\mathrm{f}_{\text {schott }}$ has nothing to do with radiation reaction but describes a reversible process (total time derivative) and depends neither on the velocity nor on the acceleration; it contains the derivative of the acceleration, $\dot{a}$.

Due to the occurrence of $\dot{a}$, the Abraham-Lorentz equation (36) suffers from (II) pre- and (III) self-accelerations. These are prima facie serious 
conceptual problems, but they melt away when the Abraham-Lorentz equation (36) is rewritten as an integro-differential equation, so that (III) self-accelerations can be disposed off as artifacts of a particular mathematical way of writing down the equation of motion (cf. Jackson 1975, 797; and R. J. Cook 1984, 1986) for tutorial elaborations. This solves problem III. Problem II is solved by imposing appropriate boundary conditions.

An extension of the Abraham-Lorentz equation (36) was derived by Dirac using energy conservation for point charges, as we mentioned above; it is generally known as the Lorentz-Dirac equation (Lorentz 1909, 4849; Dirac 1938, 155; Spohn 2004, 106-118):

$$
d_{\tau} \mathrm{p}(\tau) \approx \mathrm{f}_{\mathrm{L}}+\mathrm{f}_{\text {self }},
$$

where by definition

$$
\mathrm{f}_{\text {self }} \equiv \mathrm{f}_{\text {Schott }}+\mathrm{f}_{\text {rad }},
$$

where $f_{\text {rad }}$ is the radiation-reaction force, which signals an irreversible loss of energy and momentum and is a function of the velocity and the acceleration.

With all dependencies of the different forces in position, let us write down the three-vector part of the equation of motion:

$$
m \mathbf{a}(t) \approx \mathbf{F}_{\mathrm{L}}(\mathbf{q}(t), \mathbf{u}(t))+\mathbf{F}_{\text {Schott }}(\dot{\mathbf{a}}(t))+\mathbf{F}_{\text {rad }}(\mathbf{u}(t), \mathbf{a}(t)) .
$$

(One may justifiably wonder how Dirac's equation for point charges [37] or [39], can coincide with an equation of Abraham and Lorentz for extended charges. Well, Abraham and Lorentz obtained a power series of the velocity $\mathbf{u}$ in the electron radius, $R_{\mathrm{e}}$, the leading terms of which did not contain $R_{\mathrm{e}}$; subsequent terms were dropped, as a result of which $R_{\mathrm{e}}$ almost disappeared from the equation of motion. We say almost, because $R_{\mathrm{e}}$ still occurred in the expression for the mass, deemed of electromagnetic origin. When this mass is, however, identified with the mass $m$ in Dirac's equation, then the equations coincide. Further it deserves to be mentioned that Lorentz's derivation, which one must cut and paste from various sections of his book [1909], relied on an Ansatz of a uniformly moving charge, as did Abraham's derivation. Schott seems to have been the first to present a fully general derivation not relying on such an Ansatz [see Yaghjian 1992, Appendix B, for a streamlined presentation of Schott's derivation and historical details].)

In most cases the contribution of the radiation-reaction force $f_{\text {rad }}(38)$ is many orders of magnitude smaller than $f_{\text {schott }}$, which in turn is many orders of magnitude smaller than $f_{L}-$ whence their neglect. The LorentzDirac equation (37) also suffers from (II) pre- and (III) self-accelerations 
(see Dirac 1938, 157, 159; Jackson 1975, 784). As in the case of the Abraham-Lorentz equation, the problems II and III disappear when the Lorentz-Dirac equation is rewritten in the form of an integro-differential equation (see Ibison and Puthoff 2001).

But it also deserves to be mentioned that imposing certain boundary conditions on the original differential equation (39) eliminates all unwanted behavior (Rohrlich 1965, 168-169) and emphasizes that these conditions are not ad hoc because there are independent physical reasons for imposing them. Remember that the Cauchy problem for an equation containing $\mathbf{q}(t)$ and its first three partial time-derivatives is only well posed when these are all given at one instant of time, $\dot{\mathbf{a}}(t)$ included. Dirac (1938, 158) pointed out that when we impose the boundary condition that the particle behaves freely when it is far away from the external fields, so that $\mathbf{a}(t) \rightarrow 0$ and $\mathbf{\mathbf { a }}(t) \rightarrow 0$ for $t \rightarrow \infty$ - as we similarly do in scattering theorythen not only do we have a well-posed problem but we are also delivered from self-accelerations. The existence of solutions for the resulting Cauchy-like problem under very general conditions was proved by Hale and Stokes (1962).

A similar way to solve problems I, II, and III is to renormalize the mass $m$ occurring in the Lorentz-Dirac equation (37) and to renormalize the equation itself to the equation of motion of a free particle in the absence of external fields. This line was pursued successfully by A. O. Barut in a series of papers (see Barut 1988, 1990, 1992). This is, to repeat, another way to solve problems I, II, and III.

A completely different way to solve problems II and III is to replace $\mathrm{f}_{\text {Schott }}$ in (38) with an entirely different term, so as to remove $\dot{\mathrm{a}}(t)$ from the equation of motion (39) altogether because $i t$ alone is responsible for (II) pre- and (III) self-accelerations. For example, T. C. Mo and C. H. Papas argued that the only field that can accelerate a particle and therefore make it radiate is the external field, so that $\mathrm{f}_{\text {self }}$ should be expressible in terms of these fields and the particle kinematics. This leads to the Mo-Papas equation, which evokes neither (II) pre- nor (III) self-accelerations and whose solutions for typical problems where self-force effects occur differ from those of the Lorentz-Dirac equation. These differences lie far beyond what is experimentally accessible (see Mo and Papas 1971). Shen (1972, 3040) erroneously pronounced these two equations therefore "physically indistinguishable" - erroneously, because the correct statement is that all currently available evidence underdetermines the choice between the obviously physically nonequivalent Lorentz-Dirac and Mo-Pappas equations. The Mo-Papas equation has nevertheless not really caught on, because, for one reason, in contrast to the Lorentz-Dirac equation, it is not (approximately) derived from "first principles," that is, the postulates of 
CED, in spite of the fact that it is an impeccable instance of the NewtonMinkowski equation (4).

Recently, M. Marino (2002) has found a way to avoid "mass-renormalisation" altogether, by redefining in a systematic way divergent integrals and limits appearing in the basic equations of CED. Marino's procedure leads to a finite expression for the total electromagnetic energy momentum of the system of point particles and fields, from which the Lorentz-Dirac equation (37) then is derived. Marino solves problem I, and his procedure renders problems II and III harmless in the sense that pre- and self-accelerations surface as artifacts of approximations; they fail to surface exactly.

The past decades have witnessed the rise of several renormalization programs in CED; they include scattering theory and the calculation of cross-sections in order to make comparisons between CED and the data gathered in particle accelerators. Some of these programs are, however, combined with the Lakatosian core of the Extension Program, to which we turn next.

7. The Extension Program. As we mentioned earlier, Abraham and Lorentz were the first to construct models of the electron within CED by means of a spherically symmetric spatially extended charge density $\rho: \mathbb{R}^{3} \rightarrow \mathbb{R}$; its extension can be characterized by a single parameter, radius $R_{\mathrm{e}}>0$. Then $U_{\mathrm{pot}}=\left(1 / 4 \pi \epsilon_{0}\right) e^{2} / R_{\mathrm{e}}$ of the associated electric field is finite, and by equating it to $m_{\mathrm{e}} c^{2}$ one obtains the classical electron radius of $r_{\mathrm{e}} \equiv\left(1 / 4 \pi \epsilon_{0}\right) e^{2} / m_{\mathrm{e}} c^{2}$, which is in the order of magnitude of $10^{-12} \mathrm{~mm}$. This solves problem I. Unlike Abraham (who later, however, followed suit), Lorentz ab initio took into consideration that an extended charge density is deformed when described in a moving frame (Lorentz-Fitzgerald contraction). In 1906, H. Poincaré pioneered the resulting mechanical stress in the extended charge and dealt with the binding forces in the charge, necessarily present in order to prevent the charge from exploding due to the repulsive Coulomb forces in its parts. These binding forces accounted for a violation, in the models of Lorentz and Abraham, of the timehonored relation between force $\mathbf{F}$ and power $P$, namely, $\mathbf{F} \cdot \mathbf{u}=P$ (see Yaghjian 1992, 9-29). Seeming violations of energy and momentum conservation were avoided by redefining relativistic energy and momentum, suggestions which go back to E. Fermi in the 1920s (see Rohrlich 1970).

Extended charges rotate even in the absence of external torques (Thomas precessesion), and this rotational motion is governed by the Fermi-Walker transport equation. But an additional spin degree of freedom must be taken into account too when we have an extended charge distribution. All rotational degrees of freedom are jointly governed by the Bargmann-MichelTelegdi equation, which squares with the Lorentz-Dirac equation for small 
velocities and gyromagnetic ratio equal to 2 (see Spohn 2004, 119-129). Thus far, translational and rotational degrees of freedom decouple, as a result of approximations in the derivation of the equations of motion just mentioned. This changed when J. S. Nodvik (1964) published a landmark paper. Nodvik lifted the Lorentz-covariant electron model to the next plane of theoretical inquiry by coupling translational and rotational degrees of freedom; this leads to a much more complicated solution of the self-problem. Recently W. Appel and M. K.-H. Kiessling (2001, 24) have taken this program even further: by treating renormalization properly, they attain "a mathematically consistent and physically viable Lorentz electrodynamics."

In 1904, Arnold Sommerfeld also investigated extended charges and showed that a uniformly charged sphere obeys, to a good nonrelativistic approximation, a differential-difference equation of motion; this equation can be derived from the Lorentz-Dirac equation (37) by ignoring nonlinear terms of the time derivatives of $\mathbf{u}(t)$. Moniz and Sharp (1977, Section II) demonstrated that this Sommerfeld equation is free from (II) pre- and (III) self-accelerations provided the radius of the sphere is larger than $2 r_{\mathrm{e}} / 3$, where to recall $r_{\mathrm{e}}$ is the classical electron radius. (Compare Rorhlich 1997, 1053, for a summary.) Problems II and III solved once again for electrons.

The Sommerfeld equation raises the question whether there is a fully relativistic differential-difference equation of motion that reduces to the Lorentz-Dirac equation in the point-particle limit and to the Sommerfeld equation in the nonrelativistic limit. P. Caldirola $(1956,307)$ answered in the affirmative and conjectured an equation of motion for a charged sphere that demonstrably possesses the mentioned properties:

$$
\mathrm{f}_{\mathrm{L}}^{\alpha} \approx-\frac{m \kappa}{\tau_{\mathrm{e}}}\left(\mathrm{u}^{\alpha}\left(\tau-\tau_{\mathrm{e}}\right)+\frac{1}{c^{2}} \mathrm{u}^{\alpha}(\tau) \mathrm{u}^{\beta}(\tau) \mathrm{u}_{\beta}\left(\tau-\tau_{\mathrm{e}}\right)\right),
$$

where $\tau_{\mathrm{e}}=2 r_{\mathrm{e}} / c$ and $\kappa$ is some constant to get the units right. But Caldirola also demonstrated that equation (40) does not give rise to (II) pre- and (III) self-accelerations. The question how to derive equation (40) remained open for more than 30 years.

The canonical monograph reviewing these and more theoretical investigations into the classical-electrodynamical behavior of electric charges until the mid-1960s was Rohrlich's (1965). The revised edition of this monograph had hardly appeared in 1990 when another monograph appeared on the subject written by the electrical engineer A. D. Yaghjian (1992). Yaghjian derived equation (40) by ignoring nonlinear terms (1992, Appendix D). Hence models of charged spheres of radius $R_{\mathrm{e}}>2 r_{\mathrm{e}} / 3$ without (II) pre- and (III) self-accelerations are in CED. Since $r_{\mathrm{e}}>2 r_{\mathrm{e}} / 3$, electrons behave decently.

More importantly, by examining the derivations of Lorentz, Abraham, 
Dirac, and others through the looking glass, Yaghjian (1992) rediscovered the frequently tacitly made assumption of slowly varying electromagnetic fields in those derivations. This assumption is now known as "the adiabatic limit." Yaghjian derived another equation of motion for a charged insulating sphere (in an external field) that does not rely on an adiabatic assumption; the binding forces, that Poincaré had posited ad hoc in order to prevent Coulomb explosion, he derived from first principles. In the resulting Yaghjian equation - another instantiation of the Newton-Minkowski equation (4) - neither (II) pre- nor (III) self-accelerations occur. They are artifacts of taking an adiabatic limit and considering a Taylor expansion of the velocity function $\mathbf{u}(t)$ beyond the domain of analyticity; when analyticity is restored by plugging in an analytic switch function in the equation of motion that turns the external force on (having a switch time not smaller than the time needed for light to cross the electron, that is, $\geqslant 2 r_{\mathrm{e}} / c$ ), pre-accelerations no longer occur where the Taylor expansion is valid, after the external force is switched on. Hence this is yet another way to solve conceptual problems I, II, and III. (See Yaghjian [1992, 6572] for details and Rohrlich [1997] for a summary.)

The adiabatic limit has been the subject of rigorous treatments, and such treatments go on to appear as we speak. The adiabatic limit really is a "space-time limit," where the time limit ensues naturally from the space limit and the speed of light via $x=c t$. One introduces a dimensionless parameter $\varepsilon>0$ and re-scales the spatial axes of a Cartesian coordinate system by a factor $1 / \varepsilon$ by writing $\phi_{\text {ext }}(\varepsilon \mathbf{r})$ and $\mathbf{A}_{\text {ext }}(\varepsilon \mathbf{r})$ for the potentials of the external electromagnetic fields. Studying slowly varying fields comes down to studying the limit $\varepsilon \downarrow 0$. In both the Abraham and the Lorentz model, $\mathrm{f}_{\text {self }}$ can be Taylor-expanded in $\varepsilon$ so as to obtain effective equations of motion, usually to order 2 in $\varepsilon$. The occurrence of (II) pre- and (III) self-accelerations is then understood as an artifact of cutting off the Taylor series in $\varepsilon$; in the full series they do not occur. This is in line with our tentative claim that, unlike the diverging potential energy for point charges, pre- and self-accelerations are not rigorous consequences of CED but artifacts of approximative solutions of C-Problems. Familiar results like Larmor's formula (13) appear after having taken the adiabatic limit and the point-particle limit, irrespective of the order in which the limits are taken, as it should be.

Further, the unique existence of a solution of the Cauchy problem in the semi-relativistic Abraham model was recently proved by A. Komech and H. Spohn (2000) and independently by G. Bauer and D. Dürr (2001). The velocity of the charge for $t \rightarrow \infty$ is bounded for every extended charge and its acceleration vanishes for $t \rightarrow \infty$ (see Spohn 2004, Chapter 5). Hence (III) self-accelerations do not occur in the semi-relativistic Abraham model, whence the conclusion that problem III is solved. Kiessling (1999) 
proved that energy and momentum in the Abraham model are conserved iff spin is taken into account. The unique existence of a solution of the Cauchy problem in the relativistic Lorentz model for electrons moving with uniform velocity was proved by Appel and Kiessling (2002). To the best of this author's knowledge, there is yet no fully general rigorous proof of the unique existence of a solution of the Cauchy problem for the relativistic Lorentz electron.

The references mentioned above in our sketches of the Renormalization and Extension Programs from the bird's eye point of view provide a far from exhaustive list. The lists of hundreds of references in Rohrlich (1965), Yaghjian (1992), and notably H. Spohn's recent state-of-the-art monograph (2004) bear testimony to the fact that Frisch's "serious conceptual problems" I, II, and III have been solved at various levels of sophistication and rigor that the uninitiated reader could not possibly have suspected to exist when reading Frisch's paper. Furthermore, Frisch also ignores A. Grünbaum's penetrating analyses of problems II and III (published in this very journal; cf. Grünbaum 1976; and Grünbaum and Janis 1977). This is the reason why we judge his presentation of CED to be partisan if not grossly misleading.

Finally we want to emphasize that both the Extension and the Renormalization Program include in their Lakatosian core all postulates of CED, which makes them subprograms of CED when CED is considered as a Lakatosian scientific research program. Frisch's claim that they are revisions of CED is dead wrong. These subprograms are strengthenings of CED because their cores are logically stronger than the core of CED, which consists only of the postulates listed in Section 2.

Here ends our criticism of Frisch's Inadequacy Claim.

Note Added in Proof. G. Belot (2007) has also responded critically to Frisch (2004) - this paper has only recently come to my attention (thanks to Peter Vickers of Leeds University for providing me with a copy). Belot remarkably claims (GB1) that there are two versions of Lorentz's force law: (i) one version for the total electromagnetic field and (ii) one for only the external electromagnetic field, and therefore concomitantly two versions of CED; and (GB2) Frisch has validly demonstrated that CED-(i) is inconsistent but not that CED-(ii) is inconsistent. Belot then chooses CED-(ii) as his own favorite version and asserts that it is consistent.

I disagree with both claims (GB1) and (GB2). There are no two versions of Lorentz's force law. If CED-(i) were a version of CED, then what on Earth would be the relation between the self-force and the self-field according to CED-(i)? An alternative to Lorentz's force law? There is, contra claim (GB1), in CED one and only one relation between the electromagnetic force on a charge in an electromagnetic field as a consequence of 
that field and that relation is provided by Lorentz's force law-(3) in fourvector notation and (10) in three-vector notation. Form (i) is employed in $C$-problems and form (ii) in $A$ - and $B$-problems of CED. Next, if Frisch had stuck to (i), then he would not have arrived at a contradiction, contra claim (GB2), because then the self-field would be ignored always and everywhere and there would never and nowhere be any emitted radiation by the moving charge. Frisch's actual argument remains fallacious, no matter what alternative versions of CED anyone imagines there to be.

\section{REFERENCES}

Appel, W., and M. K.-H. Kiessling (2001), "Mass and Spin Renormalisation in Lorentz Electrodynamics," Annals of Physics 289: 24-83.

- (2002), "Scattering and Radiation Damping in Gyroscopic Lorentz Electrodynamics," Letters of Mathematical Physics 60: 31-46.

Barut, A. O. (1988), "Lorentz-Dirac Equation and Energy Conservation for Radiating Electrons," Physics Letters A 131: 11-12.

- (1990), "Renormalization and Elimination of Pre-acceleration and Runaway-Solutions of the Lorentz-Dirac Equation," Physics Letters A 145: 387-390.

- (1992), "More on the Radiation-Reaction and Runaway Solutions in Classical Electrodynamics," Physics Letters A 169: 120-122.

Bauer, G., and D. Dürr (2001), "The Maxwell-Lorentz System of a Rigid Charge," Annales Henri Poincaré 2: 179-196.

Belot, G. (2007), "Is Classical Electrodynamics an Inconsistent Theory?" Canadian Journal of Philosophy, forthcoming.

Caldirola, P. (1956), "A New Model of Classical Electron," Nuovo Cimento Supplement 3 (10): $297-343$.

Cook, R. J. (1984), "Radiation Reaction Revisited," American Journal of Physics 52: 894 895.

- (1986), "Radiation Reaction Revisited—One More Time," American Journal of Physics 54: 569-570.

Dirac, P. A. M. (1938), "Classical Theory of Radiating Electrons," Proceedings of the Royal Society of London A 167: 148-169.

Feynman, R. P. (1964), The Feynman Lectures on Physics: Mainly Electromagnetism and Matter. Reading, MA: Addison-Wesley.

Frisch, M. (2004), "Inconsistency in Classical Electrodynamics," Philosophy of Science 71: $525-549$.

Goldstein, H. (1980), Classical Mechanics. 2nd ed. Reading, MA: Addison-Wesley.

Grünbaum, A. (1976), "Is Preacceleration of Particles in Dirac's Electrodynamics a Case of Backward Causation? The Myth of Retrocausation in Classical Electrodynamics," Philosophy of Science 43: 165-201.

Grünbaum, A., and A. I. Janis (1977), "Is There Backward Causation in Classical Electrodynamics?' Journal of Philosophy 74: 475-482.

Hale, J. K., and A. P. Stokes (1962), "Some Physical Solutions of Dirac-Type Equations," Journal of Mathematical Physics 3: 70-74.

Ibison, M., and H. E. Puthoff (2001), "Relativistic Integro-Differential Form of the LorentzDirac Equation in 3D without Runaways," Journal of Physics A 34: 3421-3428.

Jackson, J. D. (1975), Classical Electrodynamics. 2nd ed. New York: Wiley \& Sons.

Kiessling, H. K.-H. (1999), "Classical Electron Theory and Conservation Laws," Physics Letters A 258: 197-204.

Komech, A., and H. Spohn (2000), "Long-Time Asymptotics for Coupled Maxwell-Lorentz Equations," Communications in Partial Differential Equations 25: 559-584.

Landau, L. D., and E. M. Lifshitz (1975), The Classical Theory of Fields. 4th ed. Oxford: Pergamon. 
Lieu, R. (1987), "Synchrotron Radiation Reaction," Journal of Physics A 20: 2405-2413.

Lorentz, H. A. (1909), The Theory of Electrons and Its Applications to the Phenomena of Light and Radiant Heat. Leipzig: Teubner.

Marino, M. (2002), "Classical Electrodynamics of Point-Charges," Annals of Physics 301: $85-127$.

Mo, T. C., and C. H. Papas (1971), "New Equation of Motion for Classical Charged Particles," Physical Review D 4: 3566-3571.

Moniz, E. J., and D. H. Sharp (1977), "Radiaction Reaction in Non-relativistic Quantum Electrodynamics," Physical Review D 15: 2850-2865.

Nodvik, J. S. (1964), "A Covariant Form of Classical Electrodynamics of Charges of Finite Extension," Annals of Physics 28: 225-319.

Pearle, P. (1977), "Absence of Radiationless Motions of Relativistically Rigid Classical Electron," Foundations of Physics 7: 931-945.

- (1978), "When Can a Classical Electron Accelerate without Radiating?" Foundations of Physics 8: 879-891.

Rohrlich, F. (1965), Classical Charged Particles: Foundations of Their Theory. Reading, MA: Addison-Wesley.

(1970), "Electromagnetic Momentum, Energy and Mass," American Journal of Physics 38: 1310-1316.

(1997), "The Dynamics of a Charged Sphere and the Electron," American Journal of Physics 65: 1051-1056.

(1999), "Classical Self-Force," Physical Review D 60: 84017-84022.

(2000), "The Self-Force and Radiation-Reaction," American Journal of Physics 68: $1109-1112$.

Schwinger, J. (1978), "Electromagnetic Mass Revisited," Foundations of Physics 13: 373 383.

Shen, C. S. (1972), "Comment on the New Equation of Motion for Classical Charged Particles," Physical Review D 6: 3039-3040.

Spohn, H. (2004), Dynamics of Charged Particles and Their Radiation Field. Cambridge: Cambridge University Press.

Suppes, P. (2003), Representation and Invariance of Scientific Structures. Stanford, CA: CSLI Publications.

Yaghjian, A. D. (1992), Relativistic Dynamics of a Charged Sphere: Updating the LorentzAbraham Model. Berlin: Springer-Verlag. 